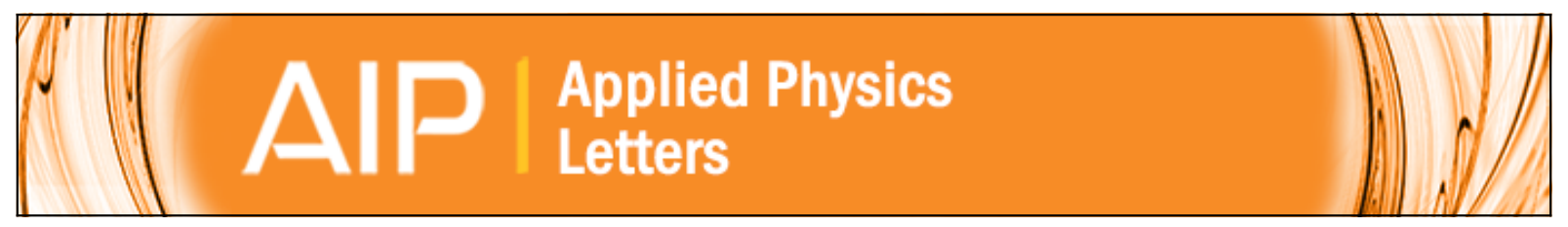

\title{
Effects of geometry and doping on the operation of molecular transistors
}

Zhongqin Yang, Norton D. Lang, and Massimiliano Di Ventra

Citation: Applied Physics Letters 82, 1938 (2003); doi: 10.1063/1.1563737

View online: http://dx.doi.org/10.1063/1.1563737

View Table of Contents: http://scitation.aip.org/content/aip/journal/apl/82/12?ver=pdfcov

Published by the AIP Publishing

\section{Articles you may be interested in}

Molecular transistors based on BDT-type molecular bridges

J. Chem. Phys. 129, 154112 (2008); 10.1063/1.2996360

First-principles investigation of the asymmetric contact effect on current-voltage characteristics of a molecular device

J. Chem. Phys. 128, 044711 (2008); 10.1063/1.2814247

Effects of hydrogen bonding on current-voltage characteristics of molecular junctions

J. Chem. Phys. 125, 194703 (2006); 10.1063/1.2364494

Vibronic effects in off-resonant molecular wire conduction

J. Chem. Phys. 118, 6072 (2003); 10.1063/1.1556854

The benzene molecule as a molecular resonant-tunneling transistor

Appl. Phys. Lett. 76, 3448 (2000); 10.1063/1.126673

\section{AlP Re-register for Table of Content Alerts}




\title{
Effects of geometry and doping on the operation of molecular transistors
}

\author{
Zhongqin Yang \\ Department of Physics, Virginia Polytechnic Institute and State University, Blacksburg, Virginia 24061 \\ Norton D. Lang \\ IBM Research Division, Thomas J. Watson Research Center, Yorktown Heights, New York 10598 \\ Massimiliano Di Ventra ${ }^{a}$ \\ Department of Physics, Virginia Polytechnic Institute and State University, Blacksburg, Virginia 24061
}

(Received 30 August 2002; accepted 29 January 2003)

\begin{abstract}
We report first-principles calculations of current versus gate voltage characteristics of a molecular transistor with a phenyldithiolate molecule as active element. We show that (i) when the molecule is placed in proximity to the gate electrode, current modulation and resonant tunneling can occur at very small gate voltages. This is due to the first-order perturbation of the electronic states induced by the electrostatic potential of the gate in the molecular region. Such perturbation is present even if the molecule does not have an intrinsic dipole moment. (ii) The molecular transistor can be converted from $n$-type to $p$-type by the simple co-adsorption of a single oxygen atom placed near the molecule. While the latter finding suggests that the character of molecular transistors can be easily changed by doping the electrode surfaces, it also puts severe constraints on the experimental control of such structures for molecular electronics applications. (c) 2003 American Institute of Physics. [DOI: $10.1063 / 1.1563737]$
\end{abstract}

In recent years, we have witnessed an increased interest in devices made of single molecules. ${ }^{1}$ Different functionalities and phenomena that can be useful in electronic applications such as resonant tunneling, Coulomb blockade, Kondo effect, or switching, have been demonstrated. ${ }^{1}$ The transistor is by far the most important element in electronics, but certainly the most difficult to realize at these nanometer-length scales. $^{2-5}$ Recently Di Ventra et al. have suggested that a single phenyldithiolate molecule with a third capacitive terminal (gate) should exhibit current modulation and resonant tunneling via the lowest unoccupied molecular orbital (LUMO) (i.e., $n$-type behavior). ${ }^{2}$ By symmetrically placing the molecule in a capacitor field, these authors have shown that the external gate bias can change the resistance of the molecule by more than an order of magnitude at a fixed source-drain voltage. ${ }^{2}$ At resonant tunneling, conductances of the order of the quantum of conductance $\left(2 e^{2} / h\right.$ $=77.5 \mu \mathrm{S}$ ) were predicted. ${ }^{2}$ With such a geometry, current amplification is induced by the second-order Stark effect, and gate voltages of about $8 \mathrm{~V}$ are required to obtain resonant tunneling and modulation. ${ }^{2}$ For actual applications, however, amplification at smaller gate voltages is desirable, as well the ability to modify the character of the transistor, say, from $n$-type to $p$-type.

In this letter, we show by means of first-principles calculations that when the molecule is placed in proximity to the gate electrode, current modulation, and resonant tunneling can occur at very small gate voltages. This result is due to the first-order perturbation of the electronic states induced by the electrostatic potential of the gate in the molecular region. Such perturbation is present even if the molecule has inversion symmetry with respect to the gate field direction.

a)Electronic mail: diventra@vt.edu
Furthermore, we show that the simple co-adsorption of an oxygen atom in proximity to the molecule changes the character of the transistor from $n$-type to $p$-type.

The system and geometry we consider are depicted in Fig. 1. The phenyldithiolate molecule is placed between source and drain gold electrodes that we model with ideal metals (jellium model). ${ }^{6,7}$ The interior electron density of the electrodes is taken to be equal to the value for metallic gold $\left(r_{s} \approx 3\right)$. The electron wave functions are computed by solving the Lippman-Schwinger equation iteratively to selfconsistency in steady state. Exchange and correlation are included in the density-functional formalism within the localdensity approximation. ${ }^{7}$ Current-induced forces have been found to have a negligible effect on the structural properties

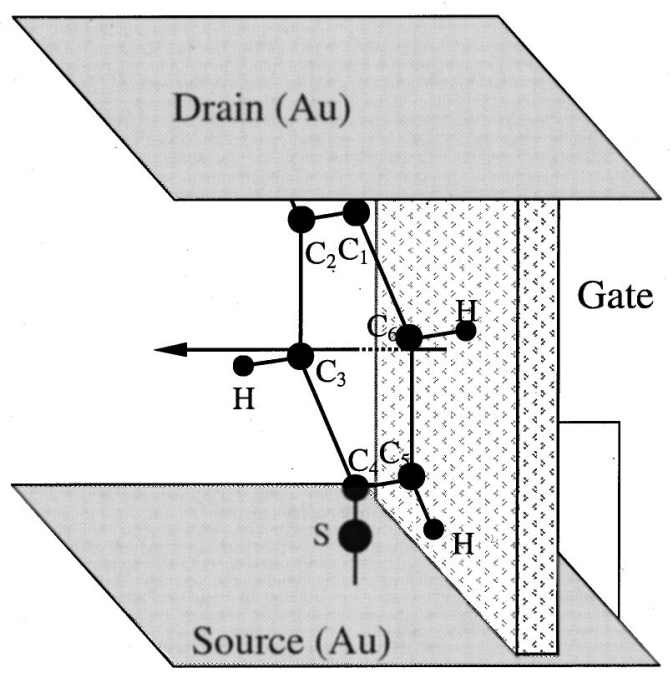

FIG. 1. Schematic of the three-terminal geometry used in the present work. The gate electrode is placed parallel to the molecular plane. 


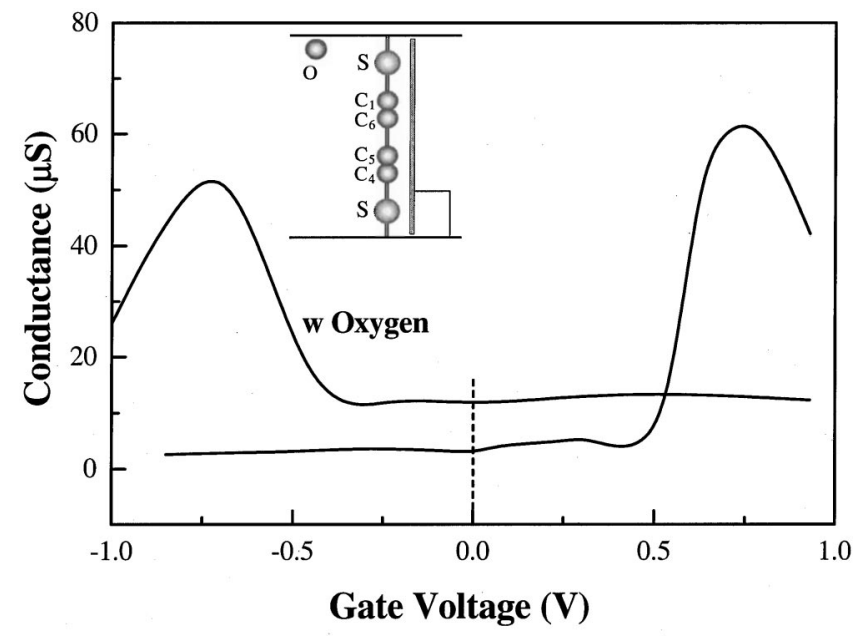

FIG. 2. Conductance as a function of the gate bias of the phenyldithiolate molecule with and without the co-adsorbed $\mathrm{O}$ atom. The source-drain voltage is $0.01 \mathrm{~V}$. The inset shows the schematic of the system with co-adsorbed oxygen.

of the molecule at small source-drain voltages. ${ }^{8}$ All atomic positions are therefore kept at their equilibrium value at zero bias. ${ }^{8}$ The gate is introduced as a capacitor field generated by two circular charged disks at a certain distance from each other. The axis of the capacitor is perpendicular to the transport direction. The molecule is placed parallel to one of the disks at a distance of 2 a.u. (see Fig. 1). ${ }^{9}$ This is a typical bonding distance of benzene molecules on insulating surfaces. ${ }^{10}$ The second disk is placed 20 a.u. away from the molecule, simulating the fact that the gate potential is zero at infinity. Note that even though the molecule does not have an intrinsic dipole moment, the present geometry introduces a term in the Hamiltonian whose expectation value on the molecular orbitals is not zero. ${ }^{11}$ The states of the whole system (molecule plus electrodes) thus experience a linear perturbation in the presence of the gate bias proportional to the strength of the gate potential in the molecular region.

The calculated conductance as a function of the gate voltage for a source-drain bias of $0.01 \mathrm{~V}$ is shown in Fig. 2 for both negative and positive gates. In the positive gate case, the conductance changes only slightly with gate voltage up to about $0.5 \mathrm{~V}$, then increases sharply to reach a maximum value close to the quantum of conductance at $0.75 \mathrm{~V}^{12}$ The conductance then decreases at larger voltages. This behavior can be rationalized by looking at the density of states (DOS) of the present system (Fig. 3). In Fig. 3 (upper curve), it is evident that for zero gate field there is a small but relatively smooth DOS through which current can flow. The LUMO lies about $1 \mathrm{eV}$ above the Fermi levels while the bonding $\pi$ orbital [corresponding to the highest occupied molecular orbital (HOMO) of the isolated molecule] is about $3 \mathrm{eV}$ below the Fermi levels (Fig. 3, upper curve). The gate shifts all states to lower energy values with increasing bias. However, it is only when the Fermi levels align with the LUMO resonance that a sudden increase of the conductance is obtained and the conductance reaches a value close to the quantum of conductance at $0.75 \mathrm{~V}$ (see Fig. 2). For gate potentials larger than this, the resonant-tunneling condition is lost and a decrease in conductance is observed (see Fig. 2).

In the negative gate case, the gate voltage shifts all states

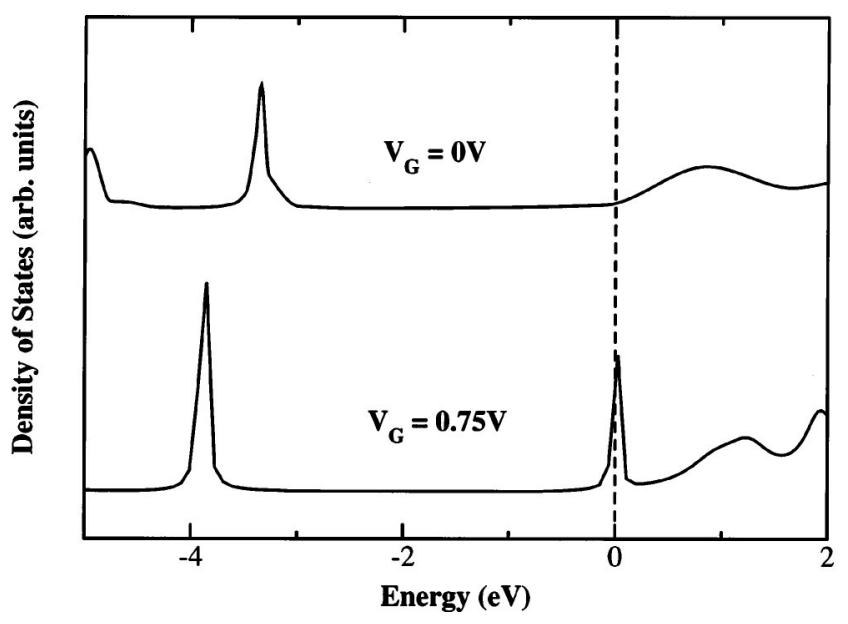

FIG. 3. Difference between the DOS of the source-drain electrodes with and without phenyldithiolate molecule in between, at zero gate voltage (upper curve) and at a gate voltage of $0.75 \mathrm{~V}$ (bottom curve). The vertical dotted line indicates the position of the left Fermi level. The right Fermi level is at $0.01 \mathrm{eV}$.

to higher energy values. The $\pi$ orbital is, however, located at about $3 \mathrm{eV}$ below the Fermi levels and the DOS is very small between the HOMO-LUMO gap. The current is thus expected to be almost constant for relatively large values of negative gate potential. This is evident in Fig. 2, where the current varies only slightly as a function of the gate field around its value at zero gate field. The small variations of the current as a function of bias can be attributed to small variations of the DOS between the HOMO-LUMO gap.

The character of the transistor considered here is $n$-type. We show that conversion to $p$-type character can be obtained by the simple co-adsorption of a single oxygen atom near the molecule/electrode junction (see inset of Fig. 2). The $\mathrm{O}$ atom is placed at 5.7 a.u. from one of the $\mathrm{S}$ atoms, at 0.2 a.u. from one of the electrode edges, and at 7.4 a.u. from the gate electrode. The current versus gate voltage characteristics change dramatically with the insertion of the oxygen atom. First, the current at zero gate voltage is a factor of 4 larger

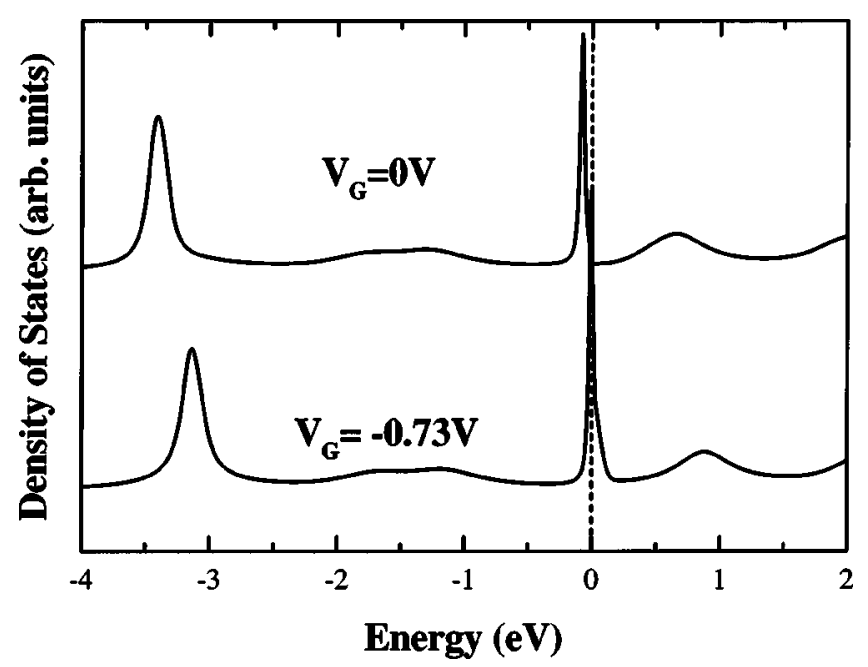

FIG. 4. Difference between the DOS of the source-drain electrodes with and without the phenyldithiolate molecule and the $\mathrm{O}$ atom, at zero gate voltage (upper curve) and at a gate voltage of $-0.73 \mathrm{~V}$ (bottom curve). The vertical dotted line indicates the position of the left Fermi leve1. The right Fermi level is at $0.01 \mathrm{eV}$. 
than the current without the oxygen (Fig. 2). ${ }^{13}$ Second, the amplification and resonant tunneling condition is obtained at negative gate voltage while, for positive voltages, the current is almost constant, giving the transistor a $p$-type character. The reason for such a dramatic change lies in the fact that the presence of the $\mathrm{O}$ atom pushes the orbitals of the molecule/ electrodes higher in energy: for this particular geometry, the HOMO is about $0.1 \mathrm{eV}$ below the Fermi level (see Fig. 4). The positive gate voltages then scan the low DOS region, giving rise to an almost constant current, while the negative gate voltages can easily push the HOMO into resonance with the lowest Fermi level. ${ }^{14}$ The shift of the molecule/electrodes energy levels to higher values due to $\mathrm{O}$ is partly due to the increase of the electrostatic potential in the molecular region, and partly to the formation of covalent bonds between oxygen and the metal electrode. ${ }^{15}$ While the latter finding suggests that the character of molecular transistors can be easily changed by doping the electrode surfaces, it also puts severe constraints on the experimental control of such structures for molecular electronics applications.

This work is supported in part by the National Science Foundation Grant Nos. DMR-01-02277 and DMR-01-33075, the Carilion Biomedical Institute, and Oak Ridge Associated Universities. Acknowledgment is also made to the Donors of The Petroleum Research Fund, administered by the American Chemical Society, for partial support of this research. The calculations reported in this letter were performed on the beowulf cluster of the Laboratory for Advanced Scientific Computing and Applications at Virginia Tech.

${ }^{1}$ See, for example, K. S. Kuok and J. C. Ellenbogen, Materials Today 5, 28 (2002); A. Aviram, M. Ratner, and V. Mujica, Molecular Electronics II (NY Academy of Sciences, New York, 2002).
${ }^{2}$ M. Di Ventra, S. T. Pantelides, and N. D. Lang, Appl. Phys. Lett. 76, 3448 (2000).

${ }^{3}$ E. G. Emberly and G. Kirczenow, J. Appl. Phys. 88, 5280 (2000).

${ }^{4}$ J. Park, A. N. Pasupathy, J. I. Goldsmith, C. Chang, Y. Yaish, J. R. Petta, M. Rinkoski, J. P. Sethna, H. D. Abruna, P. L. McEuen, and D. C. Ralph, Nature (London) 417, 722 (2002).

${ }^{5}$ W. Liang, M. P. Shores, M. Bockrath, J. R. Long, and H. Park, Nature (London) 417, 725 (2002).

${ }^{6}$ M. Di Ventra, S. T. Pantelides, and N. D. Lang, Phys. Rev. Lett. 84, 979 (2000).

${ }^{7}$ N. D. Lang, Phys. Rev. B 52, 5335 (1995); ibid. 49, 2067 (1994); M. Di Ventra and N. D. Lang, ibid. 65, 045402 (2002); Z. Yang, A. Tackett, M. Di Ventra, ibid. 66, 041405 (2002).

${ }^{8}$ M. Di Ventra, S. T. Pantelides, and N. D. Lang, Phys. Rev. Lett. 88, 046801 (2002)

${ }^{9}$ Note that, due to the particular geometry, small changes in the current versus gate voltage curve are expected if the molecular plane is placed perpendicular to the capacitor disk.

${ }^{10}$ R. A. Wolkow, G. P. Lopinski, and D. J. Moffatt, Surf. Sci. 416, L1107 (1998).

${ }^{11}$ Examples of transistor-like behavior in molecules with intrinsic dipole moment can be found in S. N. Rashkeev, M. Di Ventra, and S. T. Pantelides, Phys. Rev. B 66, 033301 (2002) and N. D. Lang, ibid. 64, 235121 (2001)

${ }^{12}$ Note that the values of the resistance at resonant tunneling are 19.2 and $16.0 \mathrm{~K} \Omega$ for the molecule/electrode system with and without the coadsorbed oxygen, respectively. This indicates that the transmission probability of the transport channel is not unity at resonance.

${ }^{13}$ N. D. Lang and Ph. Avouris (unpublished).

${ }^{14}$ Note that the gate voltage at which resonant tunneling occurs is almost the same in the presence of the $\mathrm{O}$ atom even though the HOMO is only $0.1 \mathrm{eV}$ from the left Fermi level, compared to almost $1 \mathrm{eV}$ of energy difference between the LUMO and the left Fermi level in the case without the $\mathrm{O}$ atom. The difference can be ascribed to the different screening introduced by the presence of the $\mathrm{O}$ atom.

${ }^{15} \mathrm{We}$ also tested that this effect is reduced by increasing the distance between the $\mathrm{O}$ atom and the $\mathrm{S}$ atom while keeping the O/electrode distance constant. 